\begin{tabular}{|c|c|c|c|c|c|}
\hline Revista Clio América & ISSN: 1909-941X & Vol. 11 & No. 22 & julio - diciembre de 2017 & 146 - 159 \\
\hline \multicolumn{6}{c|}{ D0I: http://10.21676/23897848.2436 } \\
\hline
\end{tabular}

\title{
Responsabilidad social empresarial en las empresas del sector minorista
}

\author{
Corporate social responsibility in the companies of the retail sector
}

\begin{abstract}
RESUMEN: El presente artículo es derivado de la investigación realizada en tiendas del sector minorista de la ciudad de Medellín, Colombia. La finalidad de la investigación fue considerar cómo la Responsabilidad Social Empresarial tiene un impacto frente a los clientes y los grupos de interés. La investigación se realizó mediante una metodología cualitativa, a través de un enfoque hermenéutico. Cabe señalar que las fuentes teóricas tienen una fundamentación sobre la gestión de la Responsabilidad Social Empresarial, aportando a una construcción empresarial de forma integral y estratégica. No obstante, se identificó que las empresas del sector minorista no están convencidas de que actuar conforme a la Responsabilidad Social Empresarial, aporta a su reputación y genera un impacto positivo frente a sus clientes y grupos de interés. Los resultados del estudio sugieren dos propuestas para implementar la responsabilidad social en las empresas de este sector; primero se plantea considerar desde la planeación estratégica de la empresa definir una política de Responsabilidad Social dentro de la organización, y la segunda, es identificar la manera en que la Responsabilidad Social aporta valor de marca y reputación frente a los clientes y demás grupos de interés.
\end{abstract}

Palabras clave: clientes; grupos de interés; Responsabilidad Social Empresarial y sector minorista.

JEL: M14, M30, L81.

ABSTRACT: his article is derived from research conducted in retail stores in the city of Medellin, Colombia. The purpose of the research was to consider how Corporate Social Responsibility has an impact on clients and interest groups. The research was carried out through a qualitative methodology, through a hermeneutical approach. It should be noted that the epistemological sources have a foundation that supports the management of Corporate Social Responsibility, contributing to a business construction in a comprehensive and strategic manner. However, it was identified that companies in the retail sector are not convinced that acting in accordance with Corporate Social Responsibility contributes to their reputation and generates a positive impact on their clients and interest groups. The results of the study suggest two proposals to implement social responsibility in companies in this sector; First, consideration is given to considering social responsibility within the strategic plan of companies, and the second is to identify the way in which Social Responsibility contributes brand value and reputation to customers and other stakeholders.

Keywords: Corporate Social Responsibility; Customers; Retail and Stakeholders.
Luis Fernando Quintero-Arango Msc. En dirección de Marketing. Universidad Católica Luis Amigo. Medellín - Colombia.

Email: luis.quinterora@amigo.edu.co

ORCID:

https://orcid.org/0000-0002-6268-065X

Tipología:

Artículo de investigación científica y tecnológica

Fecha de recibido: agosto 01 de 2017

Fecha de aceptación: noviembre 03 de 2017

Publicado en línea: noviembre 27 de 2017

Para citar este artículo: Quintero, A. L. (2017).

Responsabilidad Social Empresarial en las empresas del sector minorista. Clío América, 11(22), 146-159. Doi: $10.21676 / 23897848.2436$ 


\section{INTRODUCCIÓN}

De acuerdo con Botero (2013), actualmente el concepto de Responsabilidad Social Empresarial (RSE) es de relevancia para las empresas de la aldea global, concibiendo propuestas integrales relacionadas con su aporte al desarrollo de su actividad comercial e impacto positivo en el medio en que se desenvuelven. Para este caso, el objeto de estudio son, las tiendas del sector minorista, las cuales se consideran como aquellas que comercializan productos terminados, que los clientes compran en sus tiendas o puntos de venta; pretendiendo analizar el comportamiento de compra del consumidor moderno,; exigente y que además, valora las compañías que son socialmente responsable (Abreu y Cruz, 2011). Así mismo, estas organizaciones buscan realizar estimaciones con respecto a las necesidades y expectativas de los grupos de interés, tales como son: proveedores, accionistas, la comunidad y el propio gobierno (Martínez, 2010).

Al respecto, Restrepo (2008) explica que la RSE implica ir más allá del cumplimiento de la norma, es considerando los aspectos sociales, económicos, políticos, ambientales y culturales; siendo un concepto más integral y no obligatorio, pero si bien valorado por las compañías que buscan en la RSE una forma de diferenciarse y construir posicionamiento, suscitando que las empresas del sector logren cambiar el paradigma de solo generar utilidades y beneficio económico.

Históricamente, para abordar el tema de la Responsabilidad Social Empresarial, se ha tenido en cuenta el contexto en el que se originan las organizaciones, debido a que los trabajadores, se consideraban un objeto que solo producía -lo que primaba era el trabajo, sin contemplar su actuar como parte activa de la sociedad (Aguilera y Puerto, 2012). Navarro (2013) establece que al momento de abordar la RSE se hace una retrospectiva, con el fin de identificar que la principal preocupación solo era el interés por generar riqueza, sin considerar aspectos motivacionales y humanos frente a los empleados, así mismo, no existía preocupación por el respeto del medio ambiente y otras condiciones laborales. Este mismo autor expresa que la evolución empresarial y el paso por la revolución industrial, formó una ruptura importante para las empresas, la sociedad y los empleados, que generó cambios importantes en la forma de producir, dando paso a innovaciones que facilitaban la producción; sin embargo, las organizaciones continúan con un enfoque sesgado a los resultados sin considerar al ser humano como activo importante de la organización (Restrepo, 2008).

En el contexto actual, Boatright (2000), señala que las empresas aún continúan con un enfoque de resultados financieros, obteniendo crecimiento en sus utilidades y grandes capitales por medio de prácticas que no son bien vistas, atesorando riqueza y afectando el entorno. Por su parte, Martínez (2010), explica que la RSE inicia en las organizaciones desde su interior, valorando a sus funcionarios; desarrollando estrategias para capacitar, hacer crecer y fortalecer el vínculo entre empresa y colaborador.

Así mismo, los colaboradores de una organización deben lograr crecimiento dentro de la empresa, buscando el apoyo de la misma. Al respecto, Carroll (1999) explica que "la responsabilidad social empresarial se ha ido ligando a cuestiones como la ética empresarial, la teoría de los grupos de interés, el comportamiento social de la empresa y el concepto de ciudadanía empresarial" (p. 268). Esto lleva a considerar que el actuar de forma ética es un factor que suma a la construcción del concepto de la RSE, además que desde las lógicas empresariales se logra establecer que la organización es el reflejo de cada colaborador en su forma de actuar (Navarro, 2013).

En este sentido, Boatright (2000), manifiesta que "la ética tiene que ver con las acciones de los empleados, mientras que la responsabilidad social tiene que ver con las consecuencias de la actividad empresarial" (p. 98); está afirmación compromete a los empleados a una mayor responsabilidad de sus actuaciones y a la empresa en asumir su objeto social y empresarial de forma responsable. Por su parte, Epstein (1987) asegura que la responsabilidad social "se relaciona primordialmente con alcanzar resultados de las decisiones organizacionales respecto a temas específicos o problemas, los cuales tienen más efectos beneficiosos que adversos especialmente pertinentes a los stakeholders corporativos" (p. 104). 
Cada empresa puede implementar al interior de su organización, elementos de las diversas teorías administrativas para adoptar estrategias que puedan aportar a la reputación organizacional con sus demás grupos de interés. Vigaray (2005) analiza, que las empresas del sector minorista, tienen una RSE desde la forma en que identifican con las necesidades del mercado objetivo, guiando a la empresa a ser eficiente respecto a sus competidores y ofreciendo productos que cumplan con las regulaciones del mercado.

Según Berruezo (2003), las empresas del sector minorista tienen responsabilidad sobre su actuar desde la forma en que comercializan, exhiben, determinan el precio, el empaque, la presentación del producto y como se comunican verbal o visualmente con el cliente, buscando que este adquiera los productos de manera confiable, determinando aspectos de calidad, cantidad de los mismos, precio y garantía o respaldo sobre problemas que se puedan presentar. Así mismo, Freeman (1984) resalta, lo que son los grupos de interés dentro de la RSE, estos son quienes analizan el comportamiento de las tiendas del sector minorista; es decir, asociaciones, grupos, entidades privadas y públicas que están evaluando el actuar de las empresas en el medio, considerando tanto las repercusiones positivas como negativas de su gestión.

Por consiguiente, las empresas del sector minorista deben tener una mirada holística sobre la RSE, en donde, desde el interior de las compañías se demuestre un comportamiento que se relacione con su desempeño, con una gestión integral desde afuera; es decir, la apreciación que se genere por parte de los grupos de interés frente a las organizaciones que conforman este sector.

A continuación, en los posteriores capítulos, se desarrollan los conceptos relacionados con la RSE desde diferentes autores; así mismo, se desarrollan los temas relacionados con el punto de venta, reputación de marca y valoración por parte del cliente frente a las empresas del sector minorista. Posteriormente se aborda la Responsabilidad Social Empresarial específicamente en el sector minorista, y por último se describen los resultados y las conclusiones respectivas.

\section{Responsabilidad social empresarial}

El concepto de RSE, tiene diferentes definiciones, según los autores que soportan a la teoría sobre este concepto. Los diferentes exponentes de la RSE, tienen una concepción diferente, entre ellos se conservan algunas definiciones homogéneas, pero cada autor brinda su definición para consolidar un concepto administrativo que las empresas modernas y competitivas deben considerar dentro de su actuar organización, es así, como el sector minorista no es ajeno a la aplicación de esta teoría frente al tema propuesto. De otro lado, en los últimos años, las organizaciones han concebido que adoptar una política relacionada con la RSE tiene un impacto relevante para su marca, reputación y credibilidad en el mercado (Vigaray, 2005). Así mismo, se pueden convertir en organizaciones competitivas, valoradas por los clientes y demás grupos de interés (Botero, 2012).

De acuerdo con Volpentesta (2012), las organizaciones interpretan que los procesos de RSE, tienen un componente social, organizacional y estratégico, logrando responder de forma positiva al reto de actuar socialmente, frente a sus acciones empresariales. Es importante que el gerente o líder administrativo, lleve la impronta y transcienda en toda la organización, con el fin que el concepto de RSE sea holístico y sea aplicado por cada uno de los colaboradores.

Cabe mencionar que desde la planeación estratégica es posible definir el actuar de la empresa, es decir, establecer la misión, visión, valores y objetivos organizacionales; que incluyan estrategias articuladas a la RSE y logre permear a toda la compañía.

La Unión Europea (2011), define la responsabilidad social de las empresas como "las iniciativas voluntarias de estas, más allá de sus obligaciones legales, para lograr objetivos sociales y ambientales en su actividad cotidiana" (párr. 1). Ello implica integrar desde lo económico, social, ambiental y político, la posición que se asume frente a la RSE, enfocada en responder a lo que el mercado espera de las empresas del sector y actuar dentro de un marco regulado por normas determinadas en cada país, teniendo en cuenta el objeto social de la empresa. 
Al respecto, Friedman (1970), explica que "la única responsabilidad de la empresa hacia la sociedad es la maximización de beneficios a los accionistas dentro del marco legal y ético del país" (p. 2). Por lo tanto, es posible asumir que cada sociedad es la que determina el actuar responsable con respecto a lo que se promete entregar a los clientes, esto desde una perspectiva jurídica y ética.

Una definición más moderna es la que Guibert (2009) propone, siendo esta:

Para involucrar nuevos conceptos dentro de la RSE, podemos hablar del concepto de innovación, muy utilizado actualmente y vinculado a la competitividad de las empresas, está plenamente alineado con la RS de la empresa ya que, se ha demostrado que la RS alineada con la estrategia y la innovación afectan positivamente a la competitividad de la empresa. De hecho, no se puede promover la innovación en la empresa sin atender a aspectos directamente vinculados con la RS como son la confianza, la cooperación o los valores (p. 115).

Kramer y Porter (2006), detallan que la RSE es el valor monetario de largo plazo para las empresas e inversionistas. Las explicaciones de los anteriores autores se exponen en los trabajos "The link Between Competitive Advantage and Corporate Social Responsability" $y$ "The Competitive Advantage of Corporate Philantrophy." Al respecto, Porter y Kramer (2002) consideran que las empresas se encuentran ante una compleja disyuntiva entre la pretensión de desarrollar más actividades de RSE que gestionen el aumento del valor financiero de largo plazo de una empresa y los intereses de los inversionistas, donde se ejercen presiones para que la empresa maximice sus beneficios a corto plazo.

Por su parte, la competitividad, eficiencia y productividad, no solo se logran para mejorar los procesos productivos, reconvertir la tecnología, innovar o tener los empleados capacitados. Desde la RSE se logra impactar la calidad de vida de los colaboradores, mejorar las condiciones sobre el entorno que se hace con aportes al medio ambiente, generar procesos productivos en la zona de impacto, contribuir con empleo para las micro y pequeñas empresas, además de mantener un salario justo y una adecuada forma de contratación por parte de estas unidades productivas para ser integrales en cada una de sus actuaciones frente a la RSE (Betancur, 2010).

Para abordar el tema de la RSE y la forma de producir de las empresas generando consumo, Cortina (2002) explica que la época de compra es determinada por los sentimientos, sensaciones y percepciones del individuo, el consumismo llega a considerarse desde cada persona y sus hábitos de compra; ese excesivo consumo no diferencia cultura, edad, género, ingreso, nivel de estudios, etc., en dónde cada persona determina la suma total de la sociedad, permitiendo así, consolidar datos que determinan un excesivo consumo, en una era determinada por el consumerismo, llegando a ser considerar al individuo como irracional al momento de comprar y por los excesos innecesarios.

En este acápite, se concluye que la RSE va más allá del objeto social de la empresa, cuyo propósito es crear un tejido organizacional que impacte a los actores de la organización tales como los empleados, inversionistas, clientes y demás grupos de interés; se considera que la lógica de la RSE es ser consecuente con el actuar y la forma de ejercer las actividades comerciales.

\section{Sector minorista}

El sector minorista es considerado como aquel que vende al menudeo, teniendo un crecimiento importante en el mercado colombiano. De acuerdo con Silva (2011), los crecimientos porcentuales respecto a las ventas totales del comercio detallista en 2008, se incrementaron en un $16,7 \%$ comparativamente con el año anterior. La importancia del crecimiento en este sector es el aporte que hace al Producto Interno Bruto (PIB) del país, el impacto positivo a la generación de empleo, las oportunidades para proveedores que quieran vender a este sector, y las alianzas estratégicas con otro tipo de establecimientos que ofrecen productos y servicios complementarios.

En su boletín de prensa con respecto de la encuesta anual de comercio, el Departamento Nacional de Estadística (DANE], 2010), afirma que las personas empleadas en el sector minorista y grandes cade- 
nas, contabilizan alrededor de 98000 colaboradores para el año 2010, es decir un 5,5\% más que el año anterior. Por su parte, la revista Dinero, García (2014), explica en el libro de Retail Book, realizado por la empresa de consultoría Kantar Worldpanel, que estudia las tendencias del sector minorista y grandes superficies, que la cultura de compra de las personas que visitan estas tiendas creció un 2,5\% entre los años 2013 y 2014; así también se identificó que los consumidores visitaron las tiendas en un promedio de 20 veces más durante ese lapso de tiempo, lo que puede permitir que se definan estrategias para fidelizar a los clientes, esto con el fin de conocer sus gustos, preferencias y forma de comprar.

Lo anterior, permite analizar que el sector minorista demuestra preocupación por la generación de empleo de calidad y la disminución de los niveles de desocupación; así mismo, los clientes, proveedores y competidores, se apalancan para que las empresas detallistas sean socialmente responsables con el cliente interno y no solo miren la utilidad y el beneficio monetario, sino que se preocupan por mejorar la calidad de vida de los colaboradores y la conexión con la RSE desde el interior de la empresas (Rodríguez, 2005).

Ese lucro económico señalado previamente, tiene fundamento en ciertas referencias teóricas como Kramer y Porter (2006), Guibert (2009) y Friedman (1970), quienes expresan que desde la alta gerencia se debe considerar una ética organizacional, donde el empleado sea el articulador principal desde su desempeño laboral, con el fin de enviar un mensaje claro sobre el objeto social de la compañía y que va más allá de cumplir con ventas, utilidades y excedentes financieros. Aunque el beneficio económico es relevante, la forma de hacer conexión con el cliente y los grupos de interés es importante para que se comprenda el rol que juegan las empresas del sector minorista respecto a su actividad sobre la RSE.

En consideración con lo anterior, la actividad empresarial del sector debe construirse desde los colaboradores, con base en un código de ética, claro y enfocado en lo que busca la empresa con la RSE y el sector minorista. Para los autores señalados, es importante que se determinen algunos aspectos como la ética organizacional, el actuar del colaborador en el puesto de trabajo, el demostrar ante el cliente y los grupos de interés que se cuenta con una política de RSE sólida, lo que indique un pertinente relacionamiento endógeno y exógeno de la organización.

Para construir esa relación desde la RSE, se contempla que las empresas del sector elaboren una estrategia derivada del marketing experiencial. Al respecto López (2015) manifiesta que el marketing experiencial hace referencia a buscar experiencias positivas, que logren transmitir las cualidades del producto y lleguen hasta más allá de lo que el cliente espera; es decir, considerar transmitir por medio de los sentidos una conexión que impacte al cliente en sus estímulos sensoriales, motivando a la compra y la búsqueda de experiencias memorables.

Así es como desde las empresas del sector minorista se debe considerar tanto el consumo como el post-consumo de los clientes; es decir, identificar la forma en que la empresa brinda a los clientes, información de como se puede dar compras de manera responsable, construyendo una relación de mutuo beneficio, sin generar excesos en la compra y afectar los ingresos de las empresas. Esto podría ser de utilidad para que las empresas del sector minorista fundamenten de mejor forma la RSE en sus puntos de venta (Rodríguez, 2005).

\section{Responsabilidad social empresarial desde el sector minorista}

En el vínculo entre RSE y el sector minorista, se encuentra el aporte que hacen las empresas del sector a su entorno, sus clientes y a las personas que en algún momento requieren del apoyo en especie o dinero para superar situaciones que afectan la vida de un grupo social o un sector de la población (López, 2015).

De otro lado, se propone el siguiente interrogante para reflexionar: ¿cómo aportan las empresas del sector minorista a la RSE? Se analiza la respuesta desde el objeto social de estos negocios, en la propia dinámica empresarial. Guiber (2009) señala las oportunidades del impacto positivo de la RSE y los beneficios que puede entregar para tener una 
relación más directa con los clientes, competidores y grupos de interés, incluyendo las mismas entidades gubernamentales.

Sanclemente (cómo se citó en Wagner, Bicen y Hall, 2008), describe que:

La preocupación pública y la cobertura de los medios de comunicación acerca de la RSE en la industria de la distribución minorista se ha incrementado notablemente en los últimos años. En consecuencia, los distribuidores frecuentemente se esfuerzan por demostrar comportamientos socialmente responsables para ser percibidos como buenos ciudadanos corporativos (p. 124).

Desde la perspectiva del sector minorista y desde la operación propia de su razón social, en la revista Advanced Series (2012), se describe como el embalaje es parte fundamental de una RSE propia del sector, siendo explicado de la siguiente manera: "el embalaje es una fase crucial en la cadena de suministro del producto, en términos logísticos y comerciales, pero también ambientales" (p. 24). En relación a lo anterior, Chirinos, Fernández y Sánchez (2012) analizan el tema de lo ambiental que está relacionado con el proceso de venta, pero también se pone en evidencia que desde las óptimas condiciones del empaque, las empresas del sector minorista están haciendo un aporte al medio ambiente, buscando que ciertos materiales se utilicen, tales como el cartón, plástico, vidrio, entre otros -siendo todos ellos reciclables y reutilizables-.

Otro factor que tiene relación entre el sector minorista y la RSE, es el consumo responsable por parte de los clientes. Solís (2008) manifiesta que la empresa requiere identificar aspectos de consumo responsable, es decir, cómo se compra y qué se está comprando, identificar la forma de pago, compras por mes y otros datos que puede permitir conocer el nivel de endeudamiento de los clientes en las tiendas del sector. Por su parte, 0jeda y Jiménez (2009), proponen que para las tiendas en mención la reputación y credibilidad permean las empresas del gremio, logrando aumentar el recuerdo de la marca, posicionándose en el mercado y buscando una reputación positiva en su actuar empresarial, con el fin de promover compras socialmente respon- sables, sin excesos y endeudamientos innecesarios para los clientes.

Así mismo, las empresas del sector minorista definen su estrategia empresarial -por medio de las fundaciones creadas por ellos mismo-, favoreciendo a personas, entidades e instituciones que tengan como fin mejorar la calidad de vida de las población que se encuentren en su entorno y se identifiquen con determinadas necesidades, esto para construir un relación de beneficio para el sector y que las fundaciones cumplan su objeto social, sea en especie 0 en dinero (Navas y Londoño, 2015).

Para finalizar, es importante señalar que desde el sector minorista y su relación con la RSE -a través de las fundaciones-, una fución que cabe resaltar son las actividades de responsabilidad social que generan un impacto visible en la comunidad, tales como la entrega de alimentos para la población infantil, el apoyo que se brinda a las madres de familia, los planes de mejoramiento de vivienda y el apoyo a las escuelas del entorno, todas ellas mejorando la calidad de vida de las diferentes comunidades vulnerable (Ruíz, 2015).

\section{METODOLOGÍA}

La investigación se enfoca en las características de la hermenéutica, puesto que considera preferente el significado que los propios protagonistas dan a sus acciones mediante interpretaciones de sus pensamientos, los cuales a su vez se fundamentan en la experiencia individual subjetiva. Martínez (2009) y Tojar (2006), señalan que este enfoque se preocupa por la esencia de los fenómenos y cómo son percibidos por los sujetos, observando e interpretando el significado de los resultados, teniendo en cuenta la cultura y estilos de vida de la población objeto del estudio.

Al respecto, Taylor y Bogdan (1992) señalan que "la realidad que importa es lo que las personas perciben como importante" (p.16), buscando captar el fenómeno social en su propio escenario de acuerdo con la interpretación de cada individuo. En este sentido, Galeano (2011), argumenta que la investigación cualitativa es social y que utiliza diferentes métodos con los cuales se puede abordar el objeto 
de estudio, ya sea que se emplee la fenomenología, la hermenéutica o el interaccionismo simbólico como técnica de estudio, diversificando la forma en que se obtiene la información.

La corriente filosófica es de carácter cualitativa fenomenológica, debido a que se basa en la recolección de datos sin medición numérica, produciendo hallazgos por procedimientos no estadísticos u otros medios de cuantificación. Gran parte del análisis es interpretativo, puesto que describe fenómenos, personas, situaciones, eventos, comportamientos y actitudes de los consumidores (Bizquerra, 2009).

Según Hernández, Fernández y Baptista (2014), el enfoque inductivo presenta la forma en que un fenómeno desde lo cualitativo se puede comprender, partiendo desde lo particular a lo general en el contexto u objeto de estudio analizado. Para el estudio se tomaron como referencia las empresas del sector minorista y los clientes que visitaban los puntos de venta. El trabajo de campo se desarrolló sobre aquellos casos que son ricos en información, debido a que son inusuales o especiales en alguna forma. La lógica de este tipo de muestreo está en que del análisis de las condiciones se puede derivar información útil para comprender aspectos ocultos en las situaciones regulares (Quintana y Montgomery, 2006); Por lo tanto, se abordaron los casos de los clientes con el mayor registro de compras en cantidad y valor, los consumidores con mayor número de visitas, y los que presentaron el mayor tiempo de circulación dentro de la tienda y querían participar de forma libre en el trabajo de campo.

Se observó el comportamiento de compra en un momento del tiempo sin manipulación de información, posteriormente se realizó una observación participante, en donde se hizo un diálogo con los consumidores, permitiendo identificar el comportamiento de los clientes en el momento de la compra en las tiendas del sector minorista. Las unidades de análisis se tomaron de forma aleatoria y el diseño de esta investigación fue no experimental, de corte transversal, debido a que se recolectaron los datos en un solo momento; además, se describieron categorías que permitieron considerar su incidencia y la interrelación propia de la investigación cualitativa. Las unidades de análisis presentadas fueron: punto de venta, atención, productos que cuentan con empaques biodegradables, reutilizables o reciclables; tambien se tuvo en cuenta si las tiendas del sector minorista cuentan con procesos internos de separación de residuos sólidos, permitiendo disminuir los impactos sobre los daños ambientales.

Otra técnica utilizada fue la entrevista coloquial, Tojar (2006) explica que esta técnica es útil para grabar audio o vídeo, la cual se aplicó directamente a los sujetos participantes para lograr una información libre, espontánea, sin presiones y sesgos, con el fin de recoger la información directamente de quién está en su contexto natural. Esto fue de utilidad para analizar y evidenciar el desarrollo del proceso de compra. Cabe mencionar que el investigador presentó una actividad ética, cuyo fin fue lograr un trabajo de campo sin sesgo, válido y confiable, es decir, no ser intrusivo ni inducir a las respuestas que el investigador pretendía, las respuesta fueron naturales por parte de los clientes (Marañón, 2014). La fiabilidad se explica en la medida que se pueda replicar un estudio, para este caso los instrumentos utilizados en la investigación se pueden aplicar en contextos similares (Pérez, 2004). Según Scribano (2007) define la validez como aquello que el instrumento mide lo que pretende que se mida. Las técnicas utilizadas para el desarrollo de la investigación fueron coherentes y pertinentes, ya que permitió obtener la información que se consideró apropiada con respecto a la problemática propuesta.

La validez según Moral (2006), es definida como la correspondencia entre la forma en que el participante percibe los conceptos y la forma en que el investigador los retrata. Para ello se debe estar consciente de la capacidad e influencia que las actitudes de los investigadores pueden ejercer sobre los participantes, aislar las propias creencias y opiniones que puedan afectar la claridad en la interpretación de los datos, e incluir diversos perfiles de participantes que proporcionen diferentes puntos de vista del problema de estudio.

Para el caso de la investigación, partiendo de las técnicas propuestas, se realizó un proceso de entrevistas desde el contexto natural, es decir, las tiendas del sector minorías, de la cíudad de Medellín. Los criterios de selección de las personas se realizó por 
medio de su llegada al punto de venta, se solicitaba autorización antes de iniciar el dialogo y conocer si estaban de acuerdo con aportar para el proyecto de investigación. Así mismo, se realizaron consultas con los gerentes o supervisores de algunas de las tiendas, para identificar el tipo de clientes que compraba en la tienda, y como era el proceso de la experiencia vivida dentro de la misma.

Pérez (2004) expresa que la triangulación de las fuentes se da por los resultados obtenidos, la calidad de la información y el contraste con la teoría propuesta, así mismo, se hace referencia a las condiciones de los investigadores frente a la forma de utilizar de manera adecuada las técnicas propuestas y los métodos que se utilizaron en la investigación.

\section{RESULTADOS}

La aplicación de la RSE es determinada por actuaciones responsables, éticas, transparentes, claras y definidas desde lo que la empresa en el sector minorista demuestra ante sus clientes, competidores y grupos de interés. Por ello es importante que las empresas del sector minorista logren apropiarse del concepto de la RSE y que puedan gestionar los recursos físicos, humanos, logísticos y financieros para cumplir con el propósito de aplicación, de forma integral, ciertas propuestas de políticas organizacionales desde sus directivos hasta los empleados. Para una difusión exitosa de la aplicación de la responsabilidad social empresarial, se propone que los medios de comunicación internos, la intranet y otras herramientas físicas o tecnológicas, apoyen la gestión de la adaptación de esta nueva política de las empresas para el sector. Los colaboradores son el medio que permiten reflejar ante los clientes, proveedores, competidores y los grupos de interés tanto públicos como privados, una política enfocada en la RSE.

Los resultados encontrados en el desarrollo de la investigación contribuyen a la confirmación de estrategias que logren impactar el desempeño comercial de las empresas de este sector respecto a la RSE. Esto con la finalidad de mejorar la relación entre los clientes y los grupos de interés. Las propuestas que se plantean, buscan que la RSE sea considerada dentro del plan estratégico, además de entender cómo es que la generación de valor desde la RSE se concibe frente a los grupos de interés.

Los aspectos de la RSE se pueden convertir a corto plazo, en una herramienta innovadora que a su vez genere diferenciación, crecimiento, aportes a la sociedad y los clientes, y un ejemplo para los competidores. La RSE desde la planeación estratégica y la generación de valor se considera un factor de cambio, logrando transformaciones en su forma de actuar en el mercado, para así lograr integrar su propuesta empresarial y de RSE frente a los clientes, de tal manera que se alcance una conexión de confianza que genere una relación ganar-ganar.

A continuación se analizan los resultados encontrados, que pueden ser considerados como una propuesta para que la RSE sea incluida en la planeación estratégica de las organizaciones, dentro del contexto del sector minorista, la generación de valor; los grupos de interés y el proceso gráfico en que se puede implementar un proceso de RSE, partiendo de la empresa y su aporte de valor a los grupos de interés.

\section{La Responsabilidad Social Empresarial y su relevancia dentro de la planeación estratégica}

Cualquier organización que piense en su futuro debe desarrollar un plan de trabajo que permita establecer objetivos de corto, mediano y largo plazo, los cuales se formalicen en estrategias que aporten al logro de los objetivos. Por ello, es recomendable que la RSE tenga un espacio dentro de la planeación estratégica de las empresas del sector. Como puede observarse en la siguiente figura 1, la organización desde un contexto estratégico integra la misión, visión, valores y objetivos para gestionar actividades de las empresas hacia un cambio organizacional mediante la implementación de la RSE. Así mismo, se relacionan actuaciones para incrementar las acciones de la RSE y el plan estratégico organizacional:

- Iniciar con el diagnóstico actual de la planeación estratégica. Construir con los colaboradores el concepto de RSE desde el objeto empresarial teniendo en cuenta cómo se articula a sus actividades diarias. 
- Proponer planes que involucren a todos los colaboradores de la empresa sobre su actuar con respecto a la RSE y su relación con el cliente.

- Establecer indicadores de gestión que permitan medir la aplicación de la RSE.

- Concebir la ética como principio de respeto, valorando al cliente, los proveedores y competidores.

- Capacitar a los empleados sobre la aplicación de la RSE desde su puesto de trabajo, considerando que el actuar desde el interior de la empresa es el reflejo que el cliente percibe del exterior.

\section{Figura 1. \\ La RSE en el sector minorista y su articulación en la planeación estratégica.}

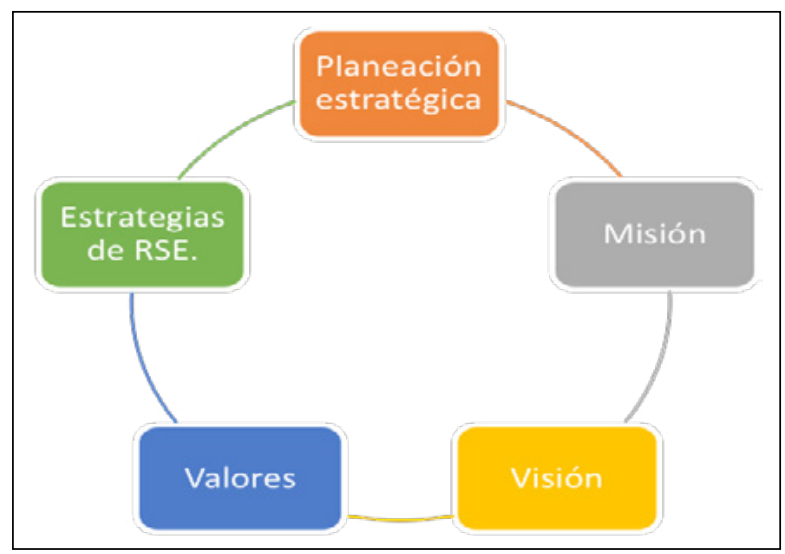

Fuente: elaboración propia.

La articulación del proceso estratégico organizacional está determinado para que la empresa considere dentro de su estructura empresarial, el desarrollo de una RSE que está permeada de forma integral en cada uno de los colaboradores, interiorizando su filosofía y comprendiendo su aplicación conceptual y práctica. Para Sarmiento (2011) la RSE es colectiva y su aplicación determina que cada individuo conforme un colectivo para que se logre la sinergia y llegue a tener un impacto tanto en el interior como en el exterior; el alcance de esta debe tener el resultado propuesto desde su planeación estratégica, así como también que cada sector de la economía tenga una identificación propia de la forma en que se puede aplicar el concepto de RSE. Lo importante es que se contemple desde la estrategia organizacional y se pueda lograr una medición de su implementación entre sus colaboradores, el entorno, los competidores y los grupos de interés.

\section{Sector minorista, generación de valor, RSE y los grupos de interés.}

El actuar de una empresa es constantemente evaluada por sus clientes, proveedores, comunidad, sociedad y competidores, por lo tanto debe estar en constante relación con sus colaboradores para que estos, desde su puesto de trabajo impacten generando valor para la empresa y sus clientes. La figura 2 explica cómo en el contexto actual, las exigencias empresariales son continuas, siendo una forma de diferenciarse y agregar valor a los consumidores desde sus procesos; además de representar una forma en que los grupos de interés evalúan su desempeño empresarial. Con lo anterior, se propone que la generación de valor considere las siguientes acciones:

a).Dejar implícito en la visión de la empresa la apuesta por la generación de valor, como una filosofía empresarial que se estructura dentro de la RSE;

b).Considerar cómo desde el plan estratégico, se puede afianzar la relación de la RSE y los grupos de interés;

c).Definir qué estrategias aportarán a la generación de valor y el impacto en la RSE;

d). Concebir cómo los informes de gestión contemplan los factores de gestión de valor y la valoración con la RSE;

e).Identificar cómo la RSE será evaluada por los grupos de interés y el plan de acción para responder a los eventos, sean estos positivos o negativos; f).Analizar cómo las estrategias de marketing apoyan a la construcción de una RSE integrada y justificada desde la generación de valor, esto con el fin de impactar a la organización en todos sus procesos. 


\section{Figura 2.}

Modelo de generación de valor, RSE y grupos de interés.

\begin{tabular}{|l|l|}
\hline \multicolumn{2}{|c|}{ Generación de valor } \\
Recurso humano \\
Responsabilidad Social Empresarial \\
$\begin{array}{l}\text { Compromiso social } \\
\text { Réblicosón con grupos externos }\end{array}$
\end{tabular}

Fuente: elaboración Propia.

Desde la generación del valor, ligado con el recurso humano, las empresas de este sector, pueden crear estrategias integrales, que logren afianzar la relación con el consumidor, considerando que sea un vínculo donde el cliente sea valorado y la empresa logra superar las expectativas que el usuario estaba esperando. Así mismo, desde la Responsabilidad Social Empresarial, se propone que el modelo de la figura 2, permita al cliente identificar que las empresas del sector minorista tengan definidas estrategias de impacto social y de un compromiso social, que articulados hacen que se logre una imagen positiva frente al usuario. El tercer aspecto relacionado con el modelo de la figura 2, es la percepción de los grupos de interés frente al comportamiento de las empresas del sector con respecto a los "evaluadores" de su actuar y la forma de relacionarse con los medios y normas establecidas para los aspecto de las RSE que deben cumplir si aplican este tipo de políticas.

El compromiso social, de las empresas se debe considerar como el impacto que se tiene por parte de la empresa hacia su entorno. Hoy la apuesta de las empresas del sector minorista, es dirigir sus esfuerzo a un desarrollo sostenible y sustentable de su objeto social, contribuyendo a un desarrollo coherente entre actividad de comercio y las estrategias definidas desde la alta gerencia; por lo anterior, se tiene una mirada más integral respecto a las acciones actuales y futuras de la empresa sobre la RSE empresarial.

La labor de las empresas del sector minorista debe ser clara, además que a través de sus puntos de venta se encuentran todos los productos que un ser humano requiere para sobrevivir y satisfacer sus necesidades básicas; por lo tanto es importante evaluar sobre cómo las empresas de este sector actúan frente a los productos pereceros o no y su destino final; al momento del vencimiento, lo anterior hace parte de la reputación y la respuesta frente a los clientes que exigen ética y transparencia al momento de comercializar este tipo de productos.

\section{CONCLUSIÓN}

La RSE se debe proyectar como una política inclusiva de la organización, es decir, desde las áreas que la conforman tales como; gerencia, finanzas, contabilidad, ventas, mercadeo, logística y servicio al cliente se convierta en una política desde el interior hacía el exterior de la empresa. La propuesta, es que los empresarios del sector minorista, tengan credibilidad y convencimiento, del aporte que las actividades de RSE generan frente a los clientes y los grupos de interés, como actividad valorada y 
generadora de reputación en el mercado. El trabajo en equipo, el aporte de los empleados, la sinergia con los proveedores y los planes que favorezcan al medio ambiente, aporten a que las empresas del sector minorista, trabajen de una manera abierta y transparente, afianzando principios éticos que se deben contemplar en el actuar diario de las actividades comerciales.

De la misma manera, el respeto por los competidores también hace parte de la RSE, con el establecimiento de acuerdos comerciales transparentes que son útiles para la toma de decisión del cliente y permitiéndole escoger bajo sus criterios donde comprar. Carroll (1999) trabaja tres escenarios para la RSE, lo enfoca desde los accionistas, es decir, la inversión que realiza una persona o entidad y espera recibir un retorno mayor; lo anterior se consigue con empresas que son transparentes en su desempeño; el otro factor es el marco regulatorio, es decir hasta donde la empresas puede moverse sin infringir las normas que desde el estado o entidades que regulan el actuar de las empresas determinan las condiciones para poder ejercer su función y trabajar de forma legal y ajustada a la realidad legal; por el último la ética, es decir los principios organizacionales que la empresa define para responder de forma honesta y clara frente a su objeto social, en este caso las empresas del sector minorista deben tener muy claro los tres factores mencionados para que puedan ejercer su función con toda transparencia.

Desde el punto de vista del empleo, el sector minorista es un buen generador, desde la creación de puestos de trabajo, ya que según el DANE (2010), para el año 2010 se logró un incrementó de 5,5 $\%$ respecto al 2009 , este factor permitió cerrar la brecha de un desempleo que en Colombia tiene tasas de dos dígitos; de otro lado, estas empresas suman al (PIB) del país, incrementando el aporte desde el sector comercio, el cual es un dinamizador de la economía desde los productos que vende; de igual forma el sector minorista se afecta cuando aparece en la economía factores como la inflación y una devaluación fuerte, estos dos indicadores impactan directamente el consumo, en este asunto es donde se debe considerar, desde la gerencia, diseñar estrategias para contrarrestar esos efectos; debido a que se puede presentar disminución de ventas afectando la rotación de inventarios, posible incremento en perdida de mercancías, incremento de las promociones para generar rotación de producto y daños especialmente en producto de consumo masivo.

Las organizaciones del sector minorista, a veces no identifican de forma clara cómo actuar frente a la RSE, pero se pueden considerar estrategias que a largo plazo lleguen dar buenos resultados; partiendo de capacitaciones para los colaboradores, generando un cambio empresarial, en la búsqueda de la innovación en los procesos.

La comprensión del concepto de RSE, permite que las empresas del sector minorista logren una relación más directa con el cliente, ya que se genera un proceso de mutuo beneficio. La RSE debe considerar un manejo transparente con el usuario, dar a conocer aspecto de apoyo y gestión frente a poblaciones vulnerables, enfatizando en que el apoyo es brindado por de la empresa y no por el cliente.

Desde el sector minorista y la conexión con la RSE, se debe proponer una articulación de políticas que permitan el vínculo entre todas las empresas que hacen parte de esta actividad comercial, buscando trabajar en la misma línea; para ello se recomienda realizar programas de capacitación entre sus empleados, diseñar proyectos que propendan por la separación de residuos sólidos, así mismo, una propuesta por enviar mensajes claros sobre sus estrategias de promoción, precio y publicidad que permitan una interpretación clara por parte del cliente.

El sector minorista debe considerar que el cliente ahora es más informado, apoyado en las redes sociales, medios de comunicación con base en la tecnología y algunos medios tradicionales pero efectivos como es el voz a voz, lo anterior, soporta la decisión de compra del cliente, analizando los comentarios y apreciaciones de otros consumidores. Ahora, el consumidor valora los temas relacionados con la protección y cuidado del medio ambiente, es decir, productos que tengan un empaque biodegradable o reciclable, de un comercio justo, donde productor y vendedor ganen, buscando tener una reputación positiva frente a los grupos de interés, proveedores y competidores. 
Así mismo, se establece que los grupos de interés, evalúan de forma constante el actuar de las tiendas del sector minorista partiendo de sus aportes al cuidado y protección del medio ambiente. Desde el contexto empresarial se valora como las empresas del sector se preocupan para que se identifiquen los beneficios que se pueden entregar al medio, clientes 0 accionistas. Los grupos de interés, no solo evalúan la empresa, sino también el actuar ético de sus empleados, estos deben ser los primeros en transmitir la RSE, es desde el interior de la empresa como se pretende aplicar y ejecutar el concepto, en la búsqueda de organizaciones que impacten de forma positiva el entorno.

Los proveedores y las empresas del sector minorista deben propender por buscar un equilibrio, donde la relación gana-gana impacte de forma positiva al cliente, para lograr un producto con condiciones de protección de marca, empaque, envase y normas de higiene y seguridad, priorizando la calidad de los productos, esto llega a ser fundamental al momento de ofrecerlos al cliente.

Desde la Responsabilidad Social Empresarial, se proponen alternativas de transformación en pro de mejorar diferenciación frente a los clientes, gestionar innovación en la experiencia de compra en la tienda, para poder lograr modificaciones positivos en el sector minorista, frente a todos los actores que están evaluando de forma constante el actuar de la empresa frente a sus clientes, proveedores y demás grupos de interés.

Esos grupos de interés analizan constantemente el actuar de las empresas del sector minorista. Conservar la reputación empresarial y una marca posicionada, tiene un impacto importante desde lo financiero y económico reflejado en resultados a mediano y largo plazo; llevando a la fidelización del cliente, valorando las estrategias que ocasionan resultados económicos positivos dentro de su sector.

Finalmente, se pretende dejar abierta la opción para que otras investigaciones de este tipo se puedan abordar desde un enfoque cuantitativo buscando valorar desde lo económico y financiero la importancia que tiene la RSE para las empresas de este sector 0 en otro que se considere pertinente, hoy es fundamental retribuir al entorno acciones sociales que construyan un equilibrio empresarial, ambiental y social. Desde el concepto de la innovación social, está tiene una relación directa con la RSE y el impacto que puedan tener frente a los competidores y los grupos de interés, determinando así una articulación entre los diferentes actores que se encuentran inmersos en este proceso empresarial y social.

\section{REFERENCIAS BIBLIOGRÁFICAS}

Abreu, J., y Cruz, J. (2011). Responsabilidad social empresas una visión integral. Revista Innovaciones de Negocios. 8(15), 71-94. Recuperado de http:// www.web.facpya.uanl.mx/rev_in/Revistas/8.1/ A4.pdf

Advanced Series (2012). El sector retail como motor de cambio hacia la sostenibilidad de la producción y el consumo [Mensaje en un blog]. Recuperado de http://vdcav.blogs.ie.edu/files/2012/12/ Sostenibilidad-Fundaci\%C3\%B3n-IE-y-ErnstYoung.pdf

Aguilera, A., y Puerto, D. (2012). Crecimiento empresarial basado en la responsabilidad social. Revista pensamiento \& gestión, 32, 1-26. Recuperado de http:// www.scielo.org.co/pdf/pege/n32/n32a02.pdf

Berruezo, J. (2003). La gestión moderna del comercio minorista. El enfoque práctico de las tiendas minoristas. Barcelona, España: Esic.

Betancur, J. (2010). La ética de la responsabilidad social empresarial. Katharsis, (9), 73-91. Recuperado de https://dialnet.unirioja.es/servlet/ articulo?codigo $=5527480$

Bizquerra, R. (2009). Metodología de la investigación educativa. Madrid, España: La Muralla.

Boatright, J. (2000). Ethics and the conduct of Business. New York, Estados Unidos: Printece Hall.

Botero, L. (2013). La globalización de la economía y la internacionalización de la empresa: una mirada en el tiempo. Revista Ciencias Estratégicas. UPB, 22(30), 203-208. Recuperado de: https://revistas.upb. edu.co/index.php/cienciasestrategicas/article/ view/2652

Carroll, A. (1999). Corporate social responsibility. Business and society, 3(38), 268-295.

Chirinos, M., Fernández, L., y Sánchez, G. (2012). Responsabilidad empresarial o empresas socialmente responsables. Revista Razón y Palabra, 17(81), 1-18. Recuperado de http://www.redalyc.org/articulo. oa?id=199524700002 
Cortina, A. (2002). Por una ética del consumo. La ciudadanía del consumidor en un mundo global. Recuperado de http://ibdigital.uib.es/greenstone/collect/cd2/ index/assoc/consumca.dir/consumcat0001.pdf

Departamento Nacional de Estadística - DANE. (2010). Encuesta anual del comercio 2009 (Boletín de Prensa EAC-2009). Recuperado de http://www.dane.gov. co/files/investigaciones/boletines/eac/bolet_eac_2009.pdf

Epstein, E. M. (1987). The corporate social policy process: Beyond business ethics, corporate responsibility, and corporate social responsiveness. California. Management Review, 29(3), 99-114.

Freeman, E. (1984). Strategic management: A stakeholder approach. Boston: Pittman.

Friedman, M. (1970). The social responsibility of businesses to increase its profits. The New York Times Magazine, 33, 1-6.

Galeano, M. (2011). Diseño de proyectos en la investigación cualitativa. Colombia: Editorial Eafit.

García, J. (12 de diciembre de 2014). Cadenas de retail pierden clientes, pero aumentan sus ventas. Dinero. Recuperado de http://www.dinero.com/ empresas/articulo/comportamiento-cadenasretail-colombia/203978

Guibert, J. (2009). Responsabilidad social empresarial. Competitividad y casos de buenas prácticas en pymes. España: Deusto.

Hernández, R., Fernández, C., y Baptista, M. (2014). Metodología de la investigación. México, D.F.: McGraw Hill.

Kramer, M. y Porter, M. (2006). Strategy and society: the link between competitive advantage and corporate social responsibility. Harvard Business Review, 78-92.

López, R. M. (2015). Persuasión a través del marketing sensorial y experiencial. Revista opción, (31). 463478. Recuperado de http://www.redalyc.org/ html/310/31045568027/

Marañón, C. (2014). La entrevista periodística como paradigma de un género dialógico: estudio del léxico en la prensa española. Revista de Comunicación, (34), 65-82. Recuperado de https://dialnet.unirioja.es/ servlet/articulo? codigo $=\mathbf{4 7 7 5 5 5 3}$

Martínez, H. (2010). Responsabilidad social y ética empresarial. Bogotá, Colombia: Ecoe.

Martínez, M. (2009). Ciencia y arte en la Metodología Cualitativa. México, D.F.: Editorial Trillas.

Moral, C. (2006). Criterios de validez en la investigación cualitativa actual. Revista de investigación educati- va, 24(1), 147-164. Recuperado de http://www. redalyc.org/pdf/2833/283321886008.pdf

Navarro, F. (2013). Responsabilidad social corporativa. Teoría y práctica. Madrid, España: Esic.

Navas, M., y Londoño, E. (2015). Las fundaciones y su concepción y gestión de la responsabilidad social. Revista saber, ciencia y libertad, 10(1), 87100. Recuperado de https://dialnet.unirioja.es/ servlet/articulo?codigo $=5295016$

0jeda, R., y Jiménez, 0. (2009). La Responsabilidad social empresarial en el sector comercial de Mérida, Yucatán, México. Revista Gestión \& Sociedad, 3(1), 74-92. Recuperado de https://revistas.lasalle.edu. co/index.php/gs/article/view/976/883

Pérez, G. (2004). Modelos de investigación cualitati$v a$ en educación social y animación sociocultural. Aplicaciones prácticas. Madrid: Narcea.

Porter, M., y Kramer, M. (2002). The competitive advantage of corporate philantrophy. Harvard Business Review, 5-20.

Quintana, A. (2006). Metodología de investigación científica cualitativa. En A. Quintana y W. Montgomery (Eds.), Psicología: Tópicos de actualidad, 47-84. Lima: UNMSM. Recuperado de http://cienciassociales. webcindario.com/PDF/Cualitativa/Inv_quintana.pdf

Restrepo, J. (2008). Apuntes sobre la responsabilidad social empresarial. Revista de derecho privado, (40), 2-10. Recuperado de http://www.redalyc.org/articulo. 0 a?id $=360033195004$

Rodríguez, M. (2005). La responsabilidad social empresarial y los consumidores. Revista de Economía Pública, Social y Cooperativa, 53, 97-109. Recuperado de http://www.redalyc.org/articulo. oa?id=17405308

Ruíz, J. (2015). Responsabilidad social empresarial universitaria: una responsabilidad interna. Revista cuadernos Latinoamericanos de administración, 12(21), 5-7. Recuperado de http://www.redalyc. org/articulo.oa?id=409643604001

Sanclemente, T. J. (2015). La responsabilidad social empresarial (RSE) de los pequeños distribuidores minoristas. Clío América, 9(17), 42-56.

Sarmiento, S. (2011). La responsabilidad social empresarial: gestión estratégica para la supervivencia de las empresas. Revista Dimensión empresarial, 9(2), 6-15. Recuperado de https://dialnet.unirioja.es/ servlet/articulo? codigo $=3965840$

Scribano, A. (2007). El proceso de investigación social cualitativo. Buenos Aíres: Prometeo. 
Silva, H. (2011). Comportamiento de las superficies de retail en Colombia. Revista Pensamiento y Gestión. (30), 3-20. Recuperado de http://www.redalyc. org/articulo.oa?id=64620756002

Solís, J. (2008). Responsabilidad social empresarial: un enfoque alternativo. Revista Análisis económico, 23(53), 227-252. Recuperado de http://www.redalyc.org/articulo.oa?id=41311449011

Taylor, S. J., y Bogdan, R. (1992). Introducción a los métodos cualitativo de investigación. Barcelona, España: Editorial Paidos.

Tojar, J. (2006). Investigación Cualitativa, Comprender y Actuar. Madrid, España: La Muralla.

Unión Europea. (2011). La responsabilidad social de las empresas (RSE) en la UE. Recuperado de http://ec.europa.eu/social/main. jsp?catId=331\&langId=es

Vigaray, M. D. (2005). Comercialización y retailing. Distribución comercial aplicada. Madrid, España: Prentice Hall.

Volpentesta, J. (2012). Las acciones sociales en empresas con responsabilidad social. Revista Visón de futuro, 16(2), 1-26. Recuperado de http://www.redalyc. org/articulo.oa?id=357935612003

Wagner, T., Bicen, P., y Hall, Z. R. (2008). The dark side of retailing: towards a scale of corporate social irresponsibility. International Journal of Retail \& Distribution Management, 36(2), 124142. Recuperado de http://www.bauer.uh.edu/ zhall/docs/ijrdmwagnerpelinhall.pdf 